\title{
Half-Simple Symmetric Venn Diagrams
}

\author{
Charles E. Killian \\ Department of Computer Science, \\ Duke University, Durham, NC \\ ckillian@cs.duke.edu \\ Carla D. Savage ${ }^{\dagger}$ \\ Department of Computer Science, \\ North Carolina State University, Raleigh, NC \\ savage@csc.ncsu.edu
}

\author{
Frank Ruskey* \\ Department of Computer Science, \\ University of Victoria, Victoria, BC \\ Mark Weston \\ Department of Computer Science, \\ University of Victoria, Victoria, BC \\ mweston@cs.uvic.ca
}

Submitted: Sep 9, 2004; Accepted: Oct 13, 2004; Published: Nov 30, 2004

Mathematics Subject Classifications: 05A10, 05A16, 06A07, 06E10

\begin{abstract}
A Venn diagram is simple if at most two curves intersect at any given point. A recent paper of Griggs, Killian, and Savage [Elec. J. Combinatorics, Research Paper 2, 2004] shows how to construct rotationally symmetric Venn diagrams for any prime number of curves. However, the resulting diagrams contain only $\left(\begin{array}{c}n \\ \lfloor n / 2\rfloor\end{array}\right)$ intersection points, whereas a simple Venn diagram contains $2^{n}-2$ intersection points. We show how to modify their construction to give symmetric Venn diagrams with asymptotically at least $2^{n-1}$ intersection points, whence the name "half-simple."
\end{abstract}

\section{Introduction}

Following Grünbaum [5], a Venn diagram for $n$ sets is a collection of $n$ simple closed curves in the plane, $\left\{\Theta_{1}, \Theta_{2}, \ldots, \Theta_{n}\right\}$, with the property that for each $S \subseteq\{1,2, \ldots, n\}$ the region

$$
\bigcap_{i \in S} \operatorname{int}\left(\Theta_{i}\right) \cap \bigcap_{i \notin S} \operatorname{ext}\left(\Theta_{i}\right)
$$

is nonempty and connected. Here $\operatorname{int}\left(\Theta_{i}\right)$ and $\operatorname{ext}\left(\Theta_{i}\right)$ denote the open interior and open exterior, respectively, of $\Theta_{i}$. A Venn diagram is simple if no 3 curves have a common point of intersection. In a Venn diagram the curves are assumed to be finitely intersecting. A Venn diagram is rotationally symmetric if there is a point $p$ such that each of the rotations

\footnotetext{
${ }^{*}$ Research supported in part by NSERC grant 0-GP-000337999

${ }^{\dagger}$ Research supported in part by NSF grant DMS-0300034 and NSA grant MDA 904-01-0-0083
} 
of $\Theta_{1}$ about $p$ by an angle of $2 \pi i / n, 0 \leq i \leq n-1$, coincides with one of the curves $\Theta_{1}, \Theta_{2}, \ldots, \Theta_{n}$. A Venn diagram is monotone if every region enclosing $k$ curves, with $0<k<n$, is adjacent to a region enclosing $k-1$ curves and a region enclosing $k+1$ curves. Monotone Venn diagrams are precisely those that can be drawn with all curves convex, as shown in [1].

Venn diagrams for $n$ sets with rotational symmetry cannot exist unless $n$ is prime (Henderson [8]) and it is shown in [4] that they do exist for all prime $n$, by a general construction. The symmetric diagrams in [4] contain $\left(\begin{array}{c}n \\ \lfloor n / 2\rfloor\end{array}\right)$ intersection points, with exactly $n$ points of intersection through which all curves pass. Such diagrams were introduced by Ruskey and Chow [9], who provided examples of them for $n=5$ and $n=7$. In [7] Hamburger constructed a symmetric Venn diagram for $n=11$ with this property.

It follows from Euler's formula $(V-E+F=2)$ that a simple Venn diagram has $2^{n}-2$ vertices. The diagrams constructed in [4], both symmetric and non-symmetric, are monotone, and among monotone Venn diagrams they contain the least number of vertices, namely:

$$
\left(\begin{array}{c}
n \\
\left\lfloor\frac{n}{2}\right\rfloor
\end{array}\right) \sim \frac{2^{n}}{\sqrt{n}} .
$$

This was shown in [2] to be minimum for monotone Venn diagrams.

Simple monotone non-symmetric diagrams exist for all $n$, but simple symmetric Venn diagrams are known only for $n=3,5$ and 7: see [9] for some examples. So, we are now motivated to ask if we can we find simple symmetric Venn diagrams for all prime $n$, or at least ones that are "more simple", where as a measure of simplicity, we use the number of vertices in the Venn diagram.

In this paper we show that for $n$ prime, we can in fact add vertices to the diagrams in [4] to produce symmetric diagrams in which the number of vertices is asymptotically at least $2^{n-1}$. The technique used in [4] makes use of some novel observations about the Greene-Kleitman symmetric chain decomposition of the Boolean lattice [3]. The paper [4] also includes a construction, for any $n$, of monotone (non-symmetric) Venn diagrams with the minimum number of vertices. We show that when the same method of "adding vertices" is applied to this case, the resulting Venn diagrams have, surprisingly, exactly $2^{n-1}$ vertices. In both cases, to accomplish this we manipulate the dual graph of the diagram, which has $2^{n}$ vertices, each of which is identified with a bitstring of length $n$. Since vertices in a graph correspond to faces in the dual, we need to modify the construction so that the dual has more faces.

The survey [9] introduced the idea of a separable vertex as a way of simplifying a Venn diagram by introducing more vertices without destroying the Venn diagram property. Adding an edge to this dual graph corresponds to separating a vertex of the Venn diagram into two vertices by pulling some curves out of the vertex. A face in the dual that cannot be subdivided into several smaller faces corresponds to a vertex that is not separable in the Venn diagram; if all vertices are not separable the diagram is termed rigid. Simple diagrams are trivially rigid. Thus the technique of adding as many faces as possible to the dual graph corresponds to separating as many vertices as possible in the Venn diagram 
until it becomes rigid.

The next section describes the Venn diagram construction from [4] which we refer to as the GKS construction, and uses an example to illustrate the general idea. In Section 3 we prove a theorem used to subdivide faces in dual graphs into many 4-faces, each of which corresponds to the intersection of two curves in the Venn diagram. In the remaining sections, this theorem is then applied to the non-symmetric monotone diagrams and then the symmetric diagrams to calculate how many new faces, and thus vertices, can be added in each case to the diagrams.

\section{The GKS Construction}

The Hasse diagram of the Boolean lattice $\mathcal{B}_{n}$, when viewed as a graph, is isomorphic to the $n$-cube, the graph whose vertices are the $n$-bit strings, with two vertices joined by an edge when they differ in only one bit. The isomorphism maps a set $S \subseteq\{1, \ldots, n\}$ to the $n$-bit string with 1 in position $i$ iff $i \in S$. The weight of a bit string is the number of ones it contains.

A subgraph of the $n$-cube is called monotone if every vertex of weight $d$ is adjacent to a vertex of weight $d+1$ (if $d<n$ ) and a vertex of weight $d-1$ (if $d>1$ ).

The GKS construction is based on the following Theorem, proved in [4].

Theorem 1 If $G$ is a plane, monotone, spanning subgraph of the $n$-cube, the dual of $G$ is a (monotone) Venn diagram.

To construct "simpler" Venn diagrams, the plan to is to get more vertices in the Venn diagram by adding more edges in the dual. We start with an intermediate construction that works for all $n$ to make the GKS construction "simpler" (but not symmetric) and then show how to modify this when $n$ is prime to make it symmetric as well. In the remainder of this section, we review the GKS construction.

In a binary string, regard each ' 1 ' as a right parenthesis and each ' 0 ' as a left parenthesis and match parentheses in the usual way. For example, in the string

$$
10010011110010
$$

the ' 1 ' bits in positions $4,7,8,9$, and 13 are matched, respectively, with the ' 0 ' bits in positions $3,5,6,2,12$. The ' 1 ' in position 10 is unmatched and the ' 0 ' bits in positions 11 and 14 are unmatched.

For every $n>0$, define the rooted tree $T_{n}$ to be the tree whose nodes are the $n$-bit strings with no unmatched 1 , and where the parent of node $x, p(x)$, is obtained from $x$ by changing the last 1 in $x$ to 0 . Note $p(x)$ is in $T_{n}$ when $x$ is, since if $x$ has no unmatched 1 , the same must be true of $p(x)$. See Figure 1 for $T_{5}$.

Given $T_{n}$, we now grow each node $x$ of $T_{n}$ into a chain $C_{x}$ of $n$-bit strings using the Greene-Kleitman successor rule [3]:

Starting with a string $x$ with no unmatched 1, change the first unmatched 0 to 1 to get its successor, $y$. Change the first unmatched 0 in $y$ to 1 (if any) to get its successor. Continue until a string with no unmatched 0 is reached. 


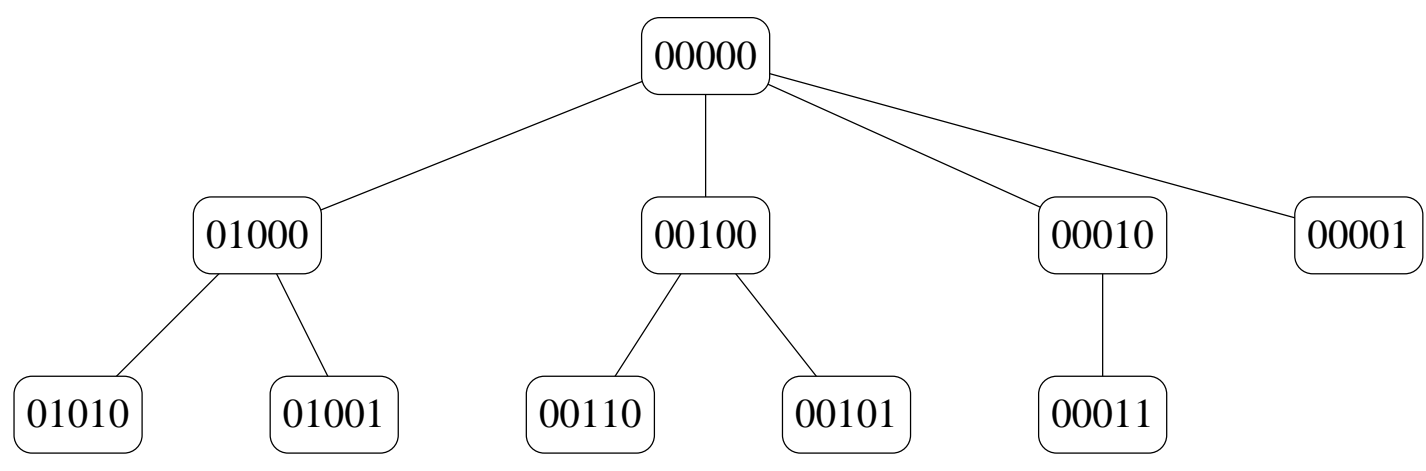

Figure 1: The rooted tree $T_{5}$ of 5 -bit strings with no unmatched 1.

As an example, by this rule node 00100 in $T_{5}$ is expanded into the chain $C_{00100}$ :

$$
C_{00100}: \quad 00100 \rightarrow 10100 \rightarrow 10110 \rightarrow 10111
$$

and the complete list of chains for nodes in $T_{5}$ is:

$$
\begin{array}{ll}
C_{00000}: & 00000 \rightarrow 10000 \rightarrow 11000 \rightarrow 11100 \rightarrow 11110 \rightarrow 11111 \\
C_{01000}: & 01000 \rightarrow 01100 \rightarrow 01110 \rightarrow 01111 \\
C_{01010}: & 01010 \rightarrow 01011 \\
C_{01001}: & 01001 \rightarrow 01101 \\
C_{00100}: & 00100 \rightarrow 10100 \rightarrow 10110 \rightarrow 10111 \\
C_{00110}: & 00110 \rightarrow 00111 \\
C_{00101}: & 00101 \rightarrow 10101 \\
C_{00010}: & 00010 \rightarrow 10010 \rightarrow 11010 \rightarrow 11011 \\
C_{00011}: & 00011 \rightarrow 10011 \\
C_{00001}: & 00001 \rightarrow 10001 \rightarrow 11001 \rightarrow 11101
\end{array}
$$

Greene and Kleitman showed in [3] that this gives a symmetric chain decomposition of the Boolean lattice. That is, (1) each of the resulting paths in the $n$-cube is a chain in $\mathcal{B}_{n}$ : each element is covered in $\mathcal{B}_{n}$ by its successor; (2) in each chain, the weights of the first and last elements sum to $n$; and (3) every $n$-bit string is in exactly one of the chains.

By definition of $T_{n}$, the first elements of $C_{x}$ and $C_{p(x)}$ differ in only one bit. In [4] it is shown that the last elements of $C_{x}$ and $C_{p(x)}$ also differ in only one bit and the chains are used to get a Venn diagram as follows.

We use the chain decomposition derived from $T_{n}$ to build a plane graph $P\left(T_{n}\right)$ that forms the dual of the final Venn diagram. Embed the chains $\left\{C_{x} \mid x \in T_{n}\right\}$ vertically in the plane, one unit apart and centered about some horizontal line, by preorder in $T_{n}$; that is, (1) for every $x, C_{x}$ precedes $C_{y}$ if $y$ is a descendant of $x$ in $T_{n}$ and (2) regarding the children of $x$ as ordered $x_{1}, \ldots, x_{t}$ for $1 \leq i \leq t-1$, chains for descendants of $x_{i}$ must appear before chains for descendants of $x_{i+1}$. (See Figure 2, ignoring the green edges). In addition, for each $x$ of positive weight, whenever $x$ is the first child of its parent $p(x)$ we include the edge joining the first elements of $C_{x}$ and $C_{p(x)}$ and the last elements of $C_{x}$ 


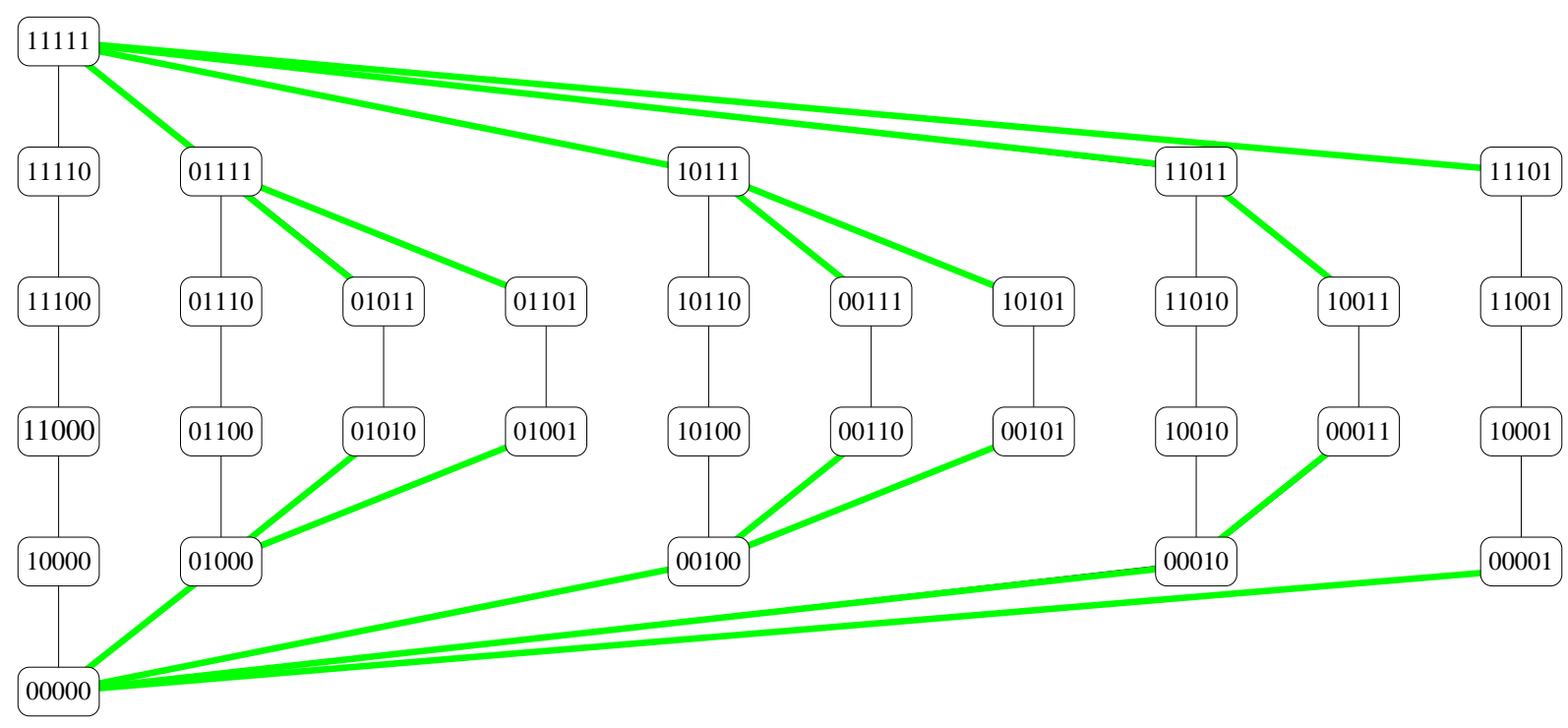

Figure 2: The plane graph $P\left(T_{5}\right)$, with attaching edges drawn in green.

and $C_{p(x)}$ (these edges must always exist, as noted above). Henceforth we refer to these edges, shown as the green edges in Figure 2, as the attaching edges for $C_{x}$.

As shown in [4], the resulting graph $P\left(T_{n}\right)$ is a plane, monotone, spanning subgraph of the $n$-cube, so by Theorem 1 , its dual is a Venn diagram. Figure 3 illustrates the process of taking the dual of $P\left(T_{4}\right)$, and Figure 4 shows the resulting Venn diagram for 4 sets. In this construction, the number of vertices in the resulting Venn diagram will be the same as the number of chains in the symmetric chain decomposition of the Boolean lattice $\mathcal{B}_{n}$, which is $\left(\begin{array}{c}n \\ \lfloor n / 2\rfloor\end{array}\right)$.

Moving now to prime $n$, the notion of block code for strings under rotation was introduced in [4] and was the key breakthrough in showing the existence of symmetric Venn diagrams for all prime $n$. Define the block code $\beta(x)$ of a binary string $x$ as follows. If $x$ starts with 0 or ends with 1 , then $\beta(x)=(\infty)$. Otherwise, $x$ can be written in the form:

$$
x=1^{a_{1}} 0^{b_{1}} 1^{a_{2}} 0^{b_{2}} \cdots 1^{a_{t}} 0^{b_{t}}
$$

for some $t>0$, where $a_{i}>0, b_{i}>0,1 \leq i \leq t$, in which case,

$$
\beta(x)=\left(a_{1}+b_{1}, a_{2}+b_{2}, \ldots, a_{t}+b_{t}\right) .
$$

The $n$ rotations of $x$, where $x$ has length $n$, are the $n$ strings reachable by applying the circular permutation $(12 \cdots n)$ to $x$. As an example, the block codes of the string 1110101100010 and all of its rotations are shown below. 


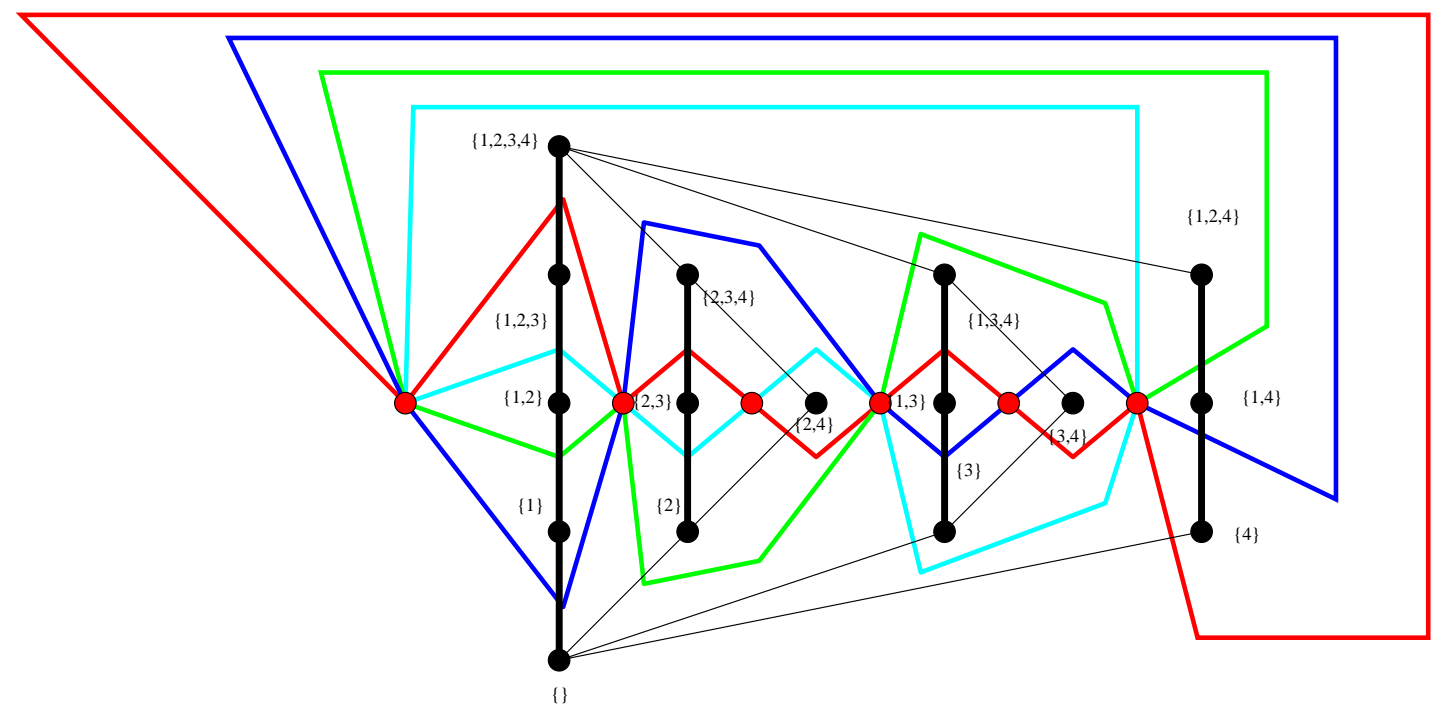

Figure 3: Construction of dual of $P\left(T_{4}\right)$.

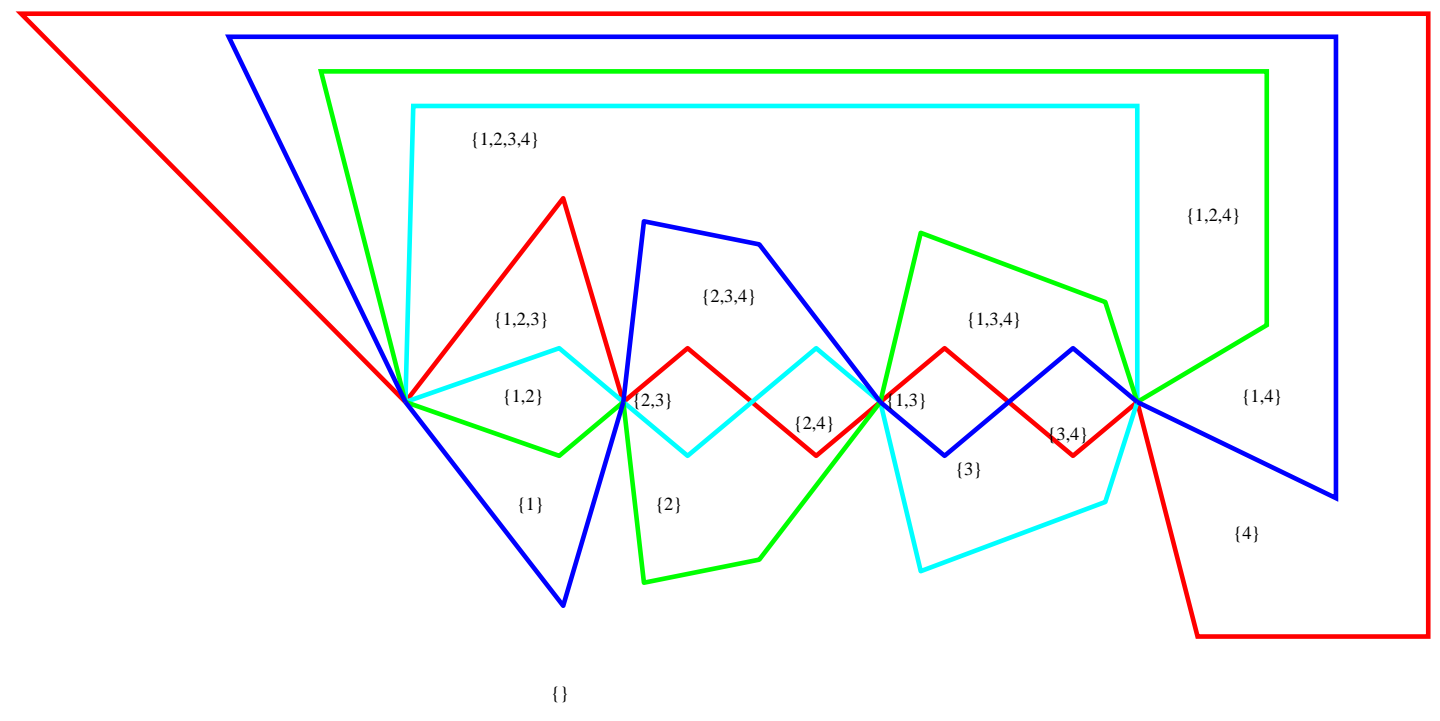

Figure 4: Venn diagram for $n=4$. 


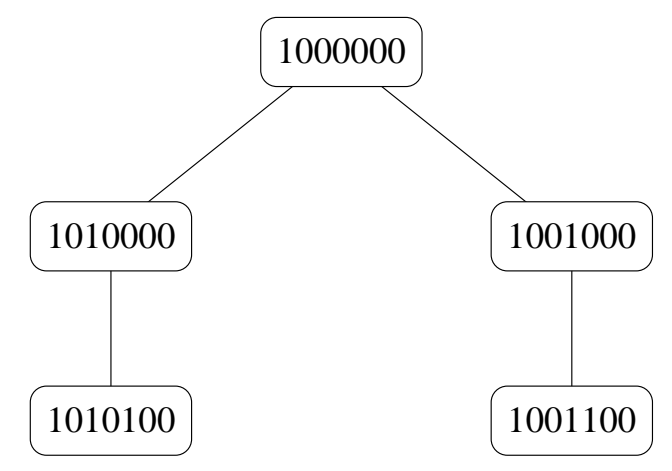

Figure 5: The rooted tree $S_{7}$ of 7 -bit rotational equivalence class representatives with one unmatched 1.

\begin{tabular}{|l|l|l|l|}
\hline bit string & block code & bit string & block code \\
\hline 1110101100010 & $(4,2,5,2)$ & 1100010111010 & $(5,2,4,2)$ \\
0111010110001 & $(\infty)$ & 0110001011101 & $(\infty)$ \\
1011101011000 & $(2,4,2,5)$ & 1011000101110 & $(2,5,2,4)$ \\
0101110101100 & $(\infty)$ & 0101100010111 & $(\infty)$ \\
0010111010110 & $(\infty)$ & 1010110001011 & $(\infty)$ \\
0001011101011 & $(\infty)$ & 1101011000101 & $(\infty)$ \\
1000101110101 & $(\infty)$ & & \\
\hline
\end{tabular}

When $n$ is prime, every $n$-bit string, other than $00 \cdots 00$ and $11 \cdots 11$, has $n$ distinct rotations. Similarly, it is shown in [4] that when $n$ is prime, no two different rotations of an $n$-bit string can have the same finite block code. Assuming that block codes are ordered lexicographically, in each equivalence class of $n$-bit strings under rotation, the unique string with minimum block code can be chosen as the representative.

For prime $n$, define the rooted tree $S_{n}$ to be the tree whose nodes are the $n$-bit strings $x$ with exactly one unmatched 1 and with $\beta(x) \leq \beta(y)$ for any rotation $y$ of $x$. Note that the unmatched 1 must appear in the leftmost position of $x$. The parent of node $x, p(x)$, is obtained from $x$ by changing the last 1 in $x$ to 0 . We note that $p(x)$ is in $S_{n}$ when $x$ is, since it is shown in [4] that (i) if $x$ has exactly one unmatched 1 , the same is true for $p(x)$ and (ii) if $\beta(x)$ is minimal under all rotations of $x$, then $\beta(p(x))$ is also. See Figure 5 for $S_{7}$.

Given $S_{n}$, we now grow each node $x$ of $S_{n}$ into a chain $C_{x}$ of $n$-bit strings using the following variation of the Greene-Kleitman construction[3]:

Start with a string $x$ which has one unmatched 1 and lexicographically smallest block code among all of its rotations (i.e., a node in $S_{n}$ ). If there is more than one unmatched 0 in $x$, change the first unmatched 0 to 1 to get its successor, $y$. If there is more than one unmatched 0 in $y$, change the first unmatched 0 in $y$ to 1 to get its successor. Continue until a string with only one unmatched 0 is reached. 
Note that a node $x$ and its successor $y$ have the same block code, so if $x$ has the minimum block code among all of its rotations, then so does $y$. Thus every element of $C_{x}$ is the (minimum block code) representative of its equivalence class under rotation.

The list of chains for nodes in $S_{7}$ is:

$$
\begin{array}{ll}
C_{1000000}: & 1000000 \rightarrow 1100000 \rightarrow 1110000 \rightarrow 1111000 \rightarrow 1111100 \rightarrow 1111110 \\
C_{1010000}: & 1010000 \rightarrow 1011000 \rightarrow 1011100 \rightarrow 1011110 \\
C_{1010100}: & 1010100 \rightarrow 1010110 \\
C_{1001000}: & 1001000 \rightarrow 1101000 \rightarrow 1101100 \rightarrow 1011110 \\
C_{1001100}: & 1001100 \rightarrow 1101100 \rightarrow 1101110 .
\end{array}
$$

It is shown in [4] that this gives a symmetric chain decomposition of the subposet of the Boolean lattice induced by the representatives of equivalence classes of $n$-bit strings under rotation (with finite block code). That is, (1) each of the resulting paths is a chain in $\mathcal{B}_{n},(2)$ each element of each chain is the (minimum block code) representative of its equivalence class under rotation, (3) in each chain, the weights of the first and last elements sum to $n$; and (4) for every $n$-bit string $x \in \mathcal{B}_{n}-\left\{0^{n}, 1^{n}\right\}$, the rotation of $x$ with lexicographically block code smallest rotation is in exactly one of the chains.

By definition of $S_{n}$, the first elements of $C_{x}$ and $C_{p(x)}$ differ in only one bit. In [4] it is shown that the last elements of $C_{x}$ and $C_{p(x)}$ also differ in only one bit and the chains are used to get a Venn diagram as follows. As before, embed the chains $\left\{C_{x} \mid x \in S_{n}\right\}$ vertically in the plane, one unit apart and centered about some horizontal line, by preorder in $S_{n}$, including the attaching edges for each $C_{x}$. As shown in [4], the resulting graph, which we call $P\left(S_{n}\right)$, is a plane, monotone, subgraph of the $n$-cube, but it only contains about $1 / n$ of the the vertices of the $n$-cube. What we need is a plane, monotone, spanning subgraph of the $n$-cube, so that by Theorem 1, its dual is a Venn diagram. In addition, we want rotational symmetry.

This is done in [4] by constructing a graph $R\left(P\left(S_{n}\right)\right)$ as follows. Start by making $n$ copies of $P\left(S_{n}\right)$, and adding $n$ copies each of the vertices $0^{n}$ and $1^{n}$ adjacent to each of the copies of $10^{n-1}$ and $1^{n-1} 0$, which start and finish the longest chain. In the $i$ th copy of $P\left(S_{n}\right)$, each vertex $x$ is replaced by the rotated vertex $\sigma^{i}(x)$, where $\sigma^{i}\left(x_{1} x_{2} \cdots x_{n}\right)=$ $x_{i+1} x_{i+2} \cdots x_{n} x_{1} \cdots x_{i}$. Each copy is embedded (symmetrically) in a $1 / n$-th pie slice in the plane, with the vertices $1^{n}$ coinciding at the center and the vertices $0^{n}$ coinciding at the point at infinity. Now, $R\left(P\left(S_{n}\right)\right)$ is a plane, monotone, spanning subgraph of the $n$-cube, so by Theorem 1, its dual is a Venn diagram. Finally, the dual of $R\left(P\left(S_{n}\right)\right)$ is constructed, preserving the symmetry. The process is illustrated in Figure 6 for $n=5$, proceeding from $P\left(S_{5}\right)$ in Figure 6a through $R\left(P\left(S_{5}\right)\right)$ in 6b to the dual of $R\left(P\left(S_{5}\right)\right)$ in $6 \mathrm{~d}$.

This construction yields rotationally symmetric Venn diagrams in which the number of vertices is the same as the number of chains in a symmetric chain decomposition of $\mathcal{B}_{n},\left(\begin{array}{c}n \\ \lfloor n / 2\rfloor\end{array}\right)$. In the next section we show a systematic way to add faces to the dual, and thereby vertices to the Venn diagram. 


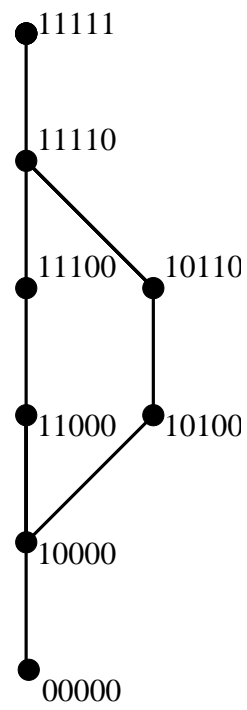

(a)

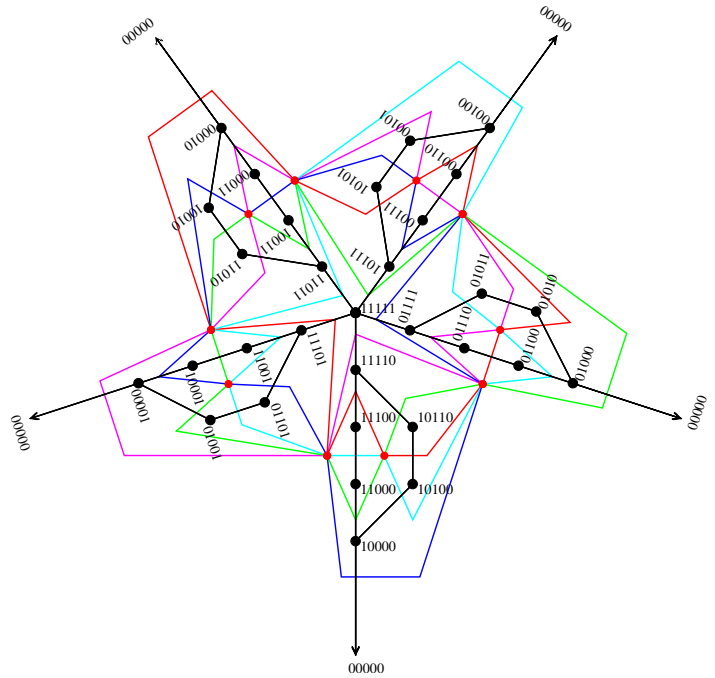

(c)

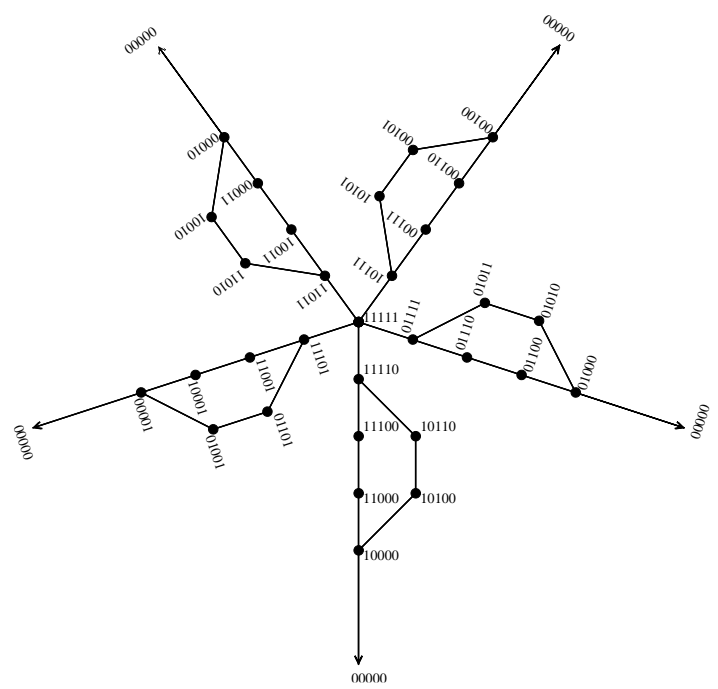

(b)

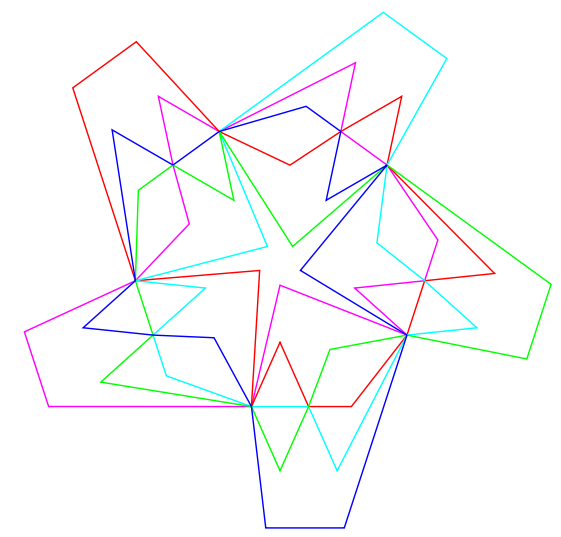

Figure 6: Building the symmetric 5-Venn diagram from $P\left(S_{5}\right)$. 


\section{Quadrangulating Faces}

In this section we show that in the chain graph, $P\left(T_{n}\right)$, any face formed by a chain and its first child can be quadrangulated (i.e. decomposed into faces bordered by 4 edges, that is, 4-faces) by adding non-crossing edges of the $n$-cube. Quadrangulating all such faces results in a plane monotone spanning subgraph $Q\left(T_{n}\right)$ of the $n$-cube. By Theorem 1 , the dual of $Q\left(T_{n}\right), Q\left(T_{n}\right)^{*}$, is a Venn diagram. Since $Q\left(T_{n}\right)$ has more faces than $P\left(T_{n}\right)$, the Venn diagram $Q\left(T_{n}\right)^{*}$ has more vertices than $P\left(T_{n}\right)^{*}$. Similarly, for prime $n$ we show that in the chain graph $P\left(S_{n}\right)$, any face formed by a chain and its first child can be quadrangulated by adding non-crossing edges of the $n$-cube and use this to get a symmetric Venn diagram with more vertices. In Sections 4 and 5 we count how many vertices are added to the Venn diagrams which result from quadrangulating these faces in $P\left(T_{n}\right)$ and $P\left(S_{n}\right)$.

Let $|C|$ denote the length of a chain $C$, that is, its number of edges.

Theorem 2 Let $w \neq 0^{n}$ be a node in $T_{n}$ and let $x$ be its parent. If chains $C_{x}$, $C_{w}$ are embedded consecutively in $P\left(T_{n}\right)$, the face bounded by the chains $C_{w}, C_{x}$, and the attaching edges of $C_{x}$ can be quadrangulated into $\left|C_{w}\right|+1$ 4-faces by adding $\left|C_{w}\right|$ edges of the $n$-cube (as shown in Figure 7).

Proof. Since $w$ is a node of $T_{n}, w$ has no unmatched 1 . Let $b$ be the position of the last 1 in $w$ and let $a$ be the position of the 0 to which it is matched. Then $w$ has the form $w=y 10^{n-b}$ and $x=y 0^{n-b+1}$. Note (i) that $a<b$ and $w$ has no unmatched 0 between $a$ and $b$ (else the 1 in position $b$ would have preferred it to the 0 in position $a$.) Also note (ii) that in $x$ position $a$ and $b$ both contain unmatched 0 bits (there is no 1 to the right of $b$ in $x$; in $w$, no 1 in $y$ matched to the 0 in position $a$, so this remains true in $x$.) Finally, note (iii) that if $U_{0}(y)$ denotes the set of positions of the unmatched 0 bits in a string $y$, then $U_{0}(x)$ is the disjoint union $U_{0}(x)=U_{0}(w) \cup\{a, b\}$.

Let $U_{0}(w)=\left\{u_{1}, u_{2}, \ldots \ldots u_{m}\right\}$, where $u_{1}<u_{2}<\cdots<u_{m}$. By (i) above, there exists $i, 0 \leq i \leq m$ such that $u_{1}<u_{2}<\cdots<u_{i}<a<b<u_{i+1}<\cdots<u_{m}$. By (iii), $U_{0}(x)=\left\{u_{1}, u_{2}, \ldots, u_{i}, a, b, u_{i+1}, u_{i+2}, \ldots, u_{m}\right\}$.

For $S \subseteq\{1, \ldots, n\}$, Define $I_{S}$ to be the $n$-bit string with $i$-th bit ' 1 ' iff $i \in S$. Then the chain grown from $w$ by the Greene-Kleitman successor rule (change first unmatched 0 to 1 ) is the chain of length $m$ :

$$
C_{w}: C_{w}(0), C_{w}(1), \ldots, C_{w}(m)
$$

where $C_{w}(0)=w$ and for $1 \leq j \leq m$,

$$
C_{w}(j)=w+I_{\left\{u_{1}, u_{2}, \ldots, u_{j}\right\}} \cdot
$$

Here '+' denotes bitwise or. The chain grown from $x$ is:

$$
C_{x}: \quad C_{x}(0), C_{x}(1), \ldots, C_{x}(m+2),
$$




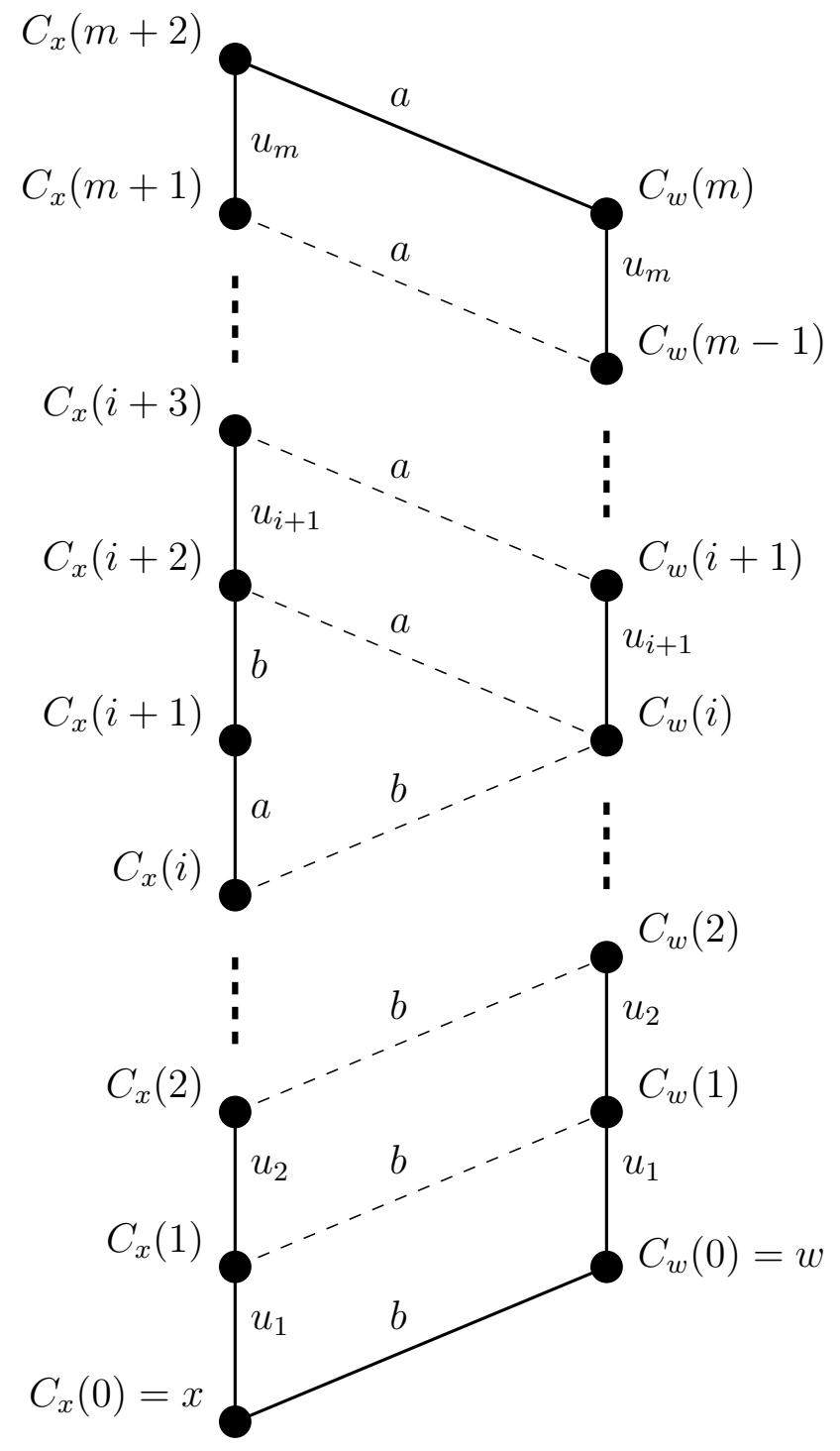

Figure 7: Quadrangulation of the face bordered by the chain $C_{w}$, its parent chain $C_{x}$, and the two attaching edges. 


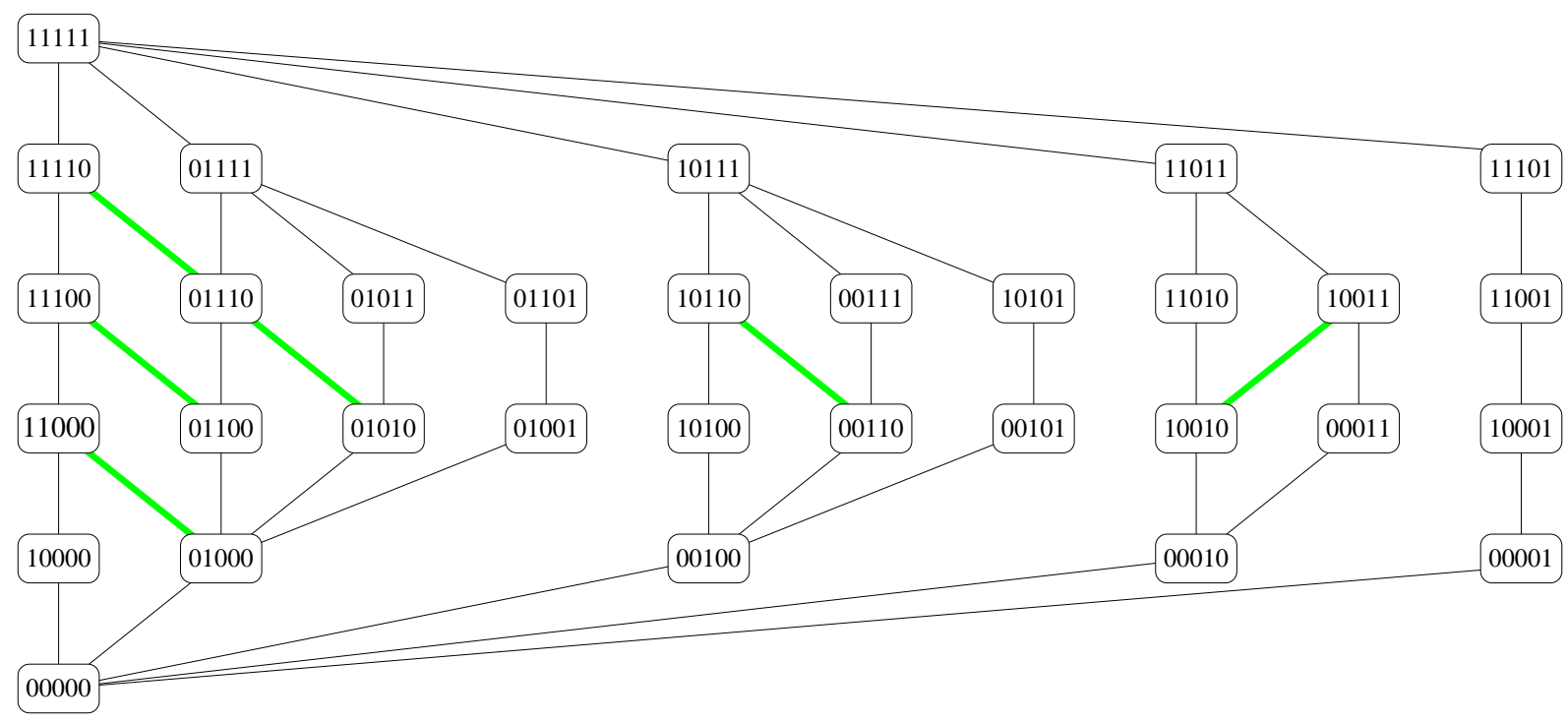

Figure 8: The plane graph $P\left(T_{5}\right)$ with quadrangulated faces.

where

$$
C_{x}(j)= \begin{cases}x & \text { if } j=0 \\ x+I_{\left\{u_{1}, u_{2}, \ldots, u_{j}\right\}} & \text { if } 1 \leq j \leq i \\ x+I_{\left\{u_{1}, u_{2}, \ldots, u_{j-1}\right\}}+I_{\{a\}} & \text { if } j=i+1 \\ x+I_{\left\{u_{1}, u_{2}, \ldots, u_{j-2}\right\}}+I_{\{a, b\}} & \text { if } i+2 \leq j \leq m+2\end{cases}
$$

Then for $0 \leq j \leq i, C_{w}(j)$ and $C_{x}(j)$ are adjacent in the $n$-cube, since they differ only in bit $b$, where $w$ and $x$ differ. For $i \leq j \leq m, C_{w}(j)$ and $C_{x}(j+2)$ are adjacent in the $n$-cube: they differ only in bit $a$, since

$$
C_{x}(j+2)=x+I_{\left\{u_{1}, u_{2}, \ldots, u_{j}\right\}}+I_{\{a, b\}}=w+I_{\left\{u_{1}, u_{2}, \ldots, u_{j}\right\}}+I_{\{a\}}=C_{w}(j)+I_{\{a\}} .
$$

Finally, if chains $C_{x}, C_{w}$ are embedded consecutively in $P\left(T_{n}\right)$, the $m$ edges

$$
\begin{gathered}
\left(C_{x}(1), C_{w}(1)\right),\left(C_{x}(2), C_{w}(2)\right), \ldots,\left(C_{x}(i), C_{w}(i)\right),\left(C_{x}(i+2), C_{w}(i)\right), \\
\left(C_{x}(i+3), C_{w}(i+1)\right), \ldots,\left(C_{x}(m+1), C_{w}(m-1)\right)
\end{gathered}
$$

can be added without crossings to create $m+1$ faces interior to the original face.

Figure 8 shows the plane graph that results if all faces of $P\left(T_{5}\right)$ bounded by chains corresponding to a node and its first child in $T_{5}$ are quadrangulated; the added edges are shaded in the figure.

We get a similar result for the plane graph $P\left(S_{n}\right)$.

Theorem 3 Let $w \neq 10^{n-1}$ be a node in $S_{n}$ and let $x$ be its parent. If chains $C_{x}, C_{w}$ are embedded consecutively in $P\left(S_{n}\right)$, the face bounded by the chains $C_{w}, C_{x}$, and the attaching edges of $C_{x}$ can be quadrangulated into $\left|C_{w}\right|+1$ 4-faces by adding $\left|C_{w}\right|$ edges of the $n$-cube. 


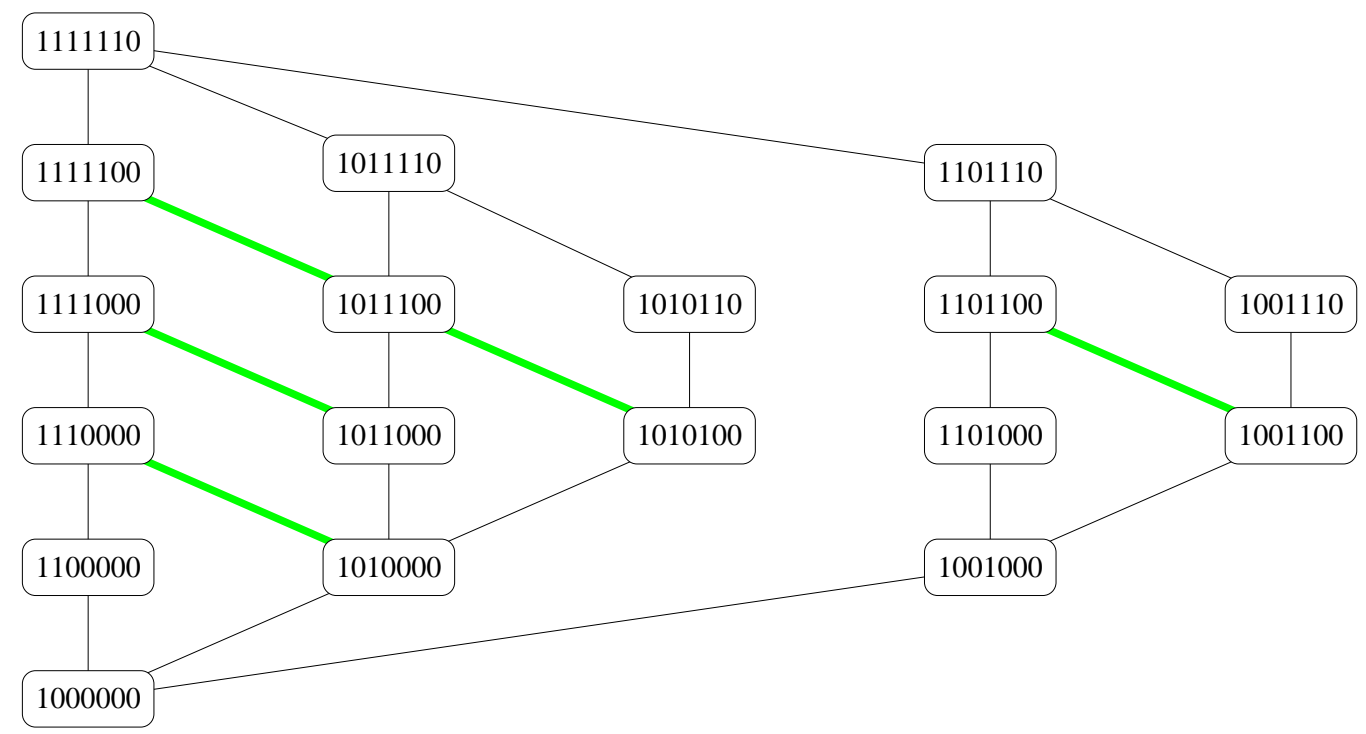

Figure 9: The plane graph $P\left(S_{7}\right)$ with quadrangulated faces.

Proof. The same proof works as for Theorem 2 after observing that for Theorem 3, if $U_{0}(w)=\left\{u_{1}, u_{2}, \ldots u_{m}\right\}$, then $C_{w}$ is the chain:

$$
C_{w}: C_{w}(0), C_{w}(1), \ldots, C_{w}(m-1) .
$$

Figure 9 shows the plane graph that results if all faces of $P\left(S_{7}\right)$ bounded by chains corresponding to a node and its first child in $S_{7}$ are quadrangulated; the added edges are shaded in the figure.

\section{Half-Simple Venn Diagrams for All $n$}

In this section we show that in $P\left(T_{n}\right)$, quadrangulating every face corresponding to a node and its first child gives a Venn diagram with $2^{n-1}$ vertices. Since quadrangulating preserves the property that $P\left(T_{n}\right)$ is a plane, monotone, spanning subgraph of the $n$-cube, the dual is still a Venn diagram. It remains only to count the number of faces added to $P\left(T_{n}\right)$ by quadrangulating.

We make the following definitions.

- Let $N_{n}$ be the total number of nodes in $T_{n}$. Each node corresponds to one chain in the symmetric chain decomposition of $\mathcal{B}_{n}$, and the number of chains is just the number of elements at the middle level of $\mathcal{B}_{n}: N_{n}=\left(\begin{array}{c}n \\ \left\lfloor\frac{n}{2}\right\rfloor\end{array}\right)$.

- Let $N_{n}(d)$ denote the number of nodes of weight $d$ in $T_{n}$. (Nodes of weight $d$ are at depth $d$ in $T_{n}$.) Then $N_{n}(d)=0$ if $d \geq\left\lfloor\frac{n}{2}\right\rfloor$. Since the chain starters at level $d$ 
in the Boolean lattice are those strings of weight $d$ which do not belong to chains started by strings of smaller weight, we have

$$
N_{n}(d)= \begin{cases}\left(\begin{array}{l}
n \\
d
\end{array}\right)-\left(\begin{array}{c}
n \\
d-1
\end{array}\right) & 0<d \leq\left\lfloor\frac{n}{2}\right\rfloor \\
1 & \text { if } d=0 \\
0 & \text { otherwise }\end{cases}
$$

Note that $\left(\begin{array}{l}n \\ d\end{array}\right)-\left(\begin{array}{c}n \\ d-1\end{array}\right)=\frac{n-2 d+1}{n-d+1}\left(\begin{array}{l}n \\ d\end{array}\right)$ is a member of the well-studied set of "ballot numbers".

- Let $\bar{L}_{n}(d)$ denote the number of nonleaves of weight $d$ in $T_{n}$.

If a node $x$ has a child in $T_{n}$, and if $w$ is its first child, then in the plane graph $P\left(T_{n}\right)$, $C_{w}$ is embedded immediately to the right of $C_{x}$. By Theorem 2, we can add $\left|C_{w}\right|=\left|C_{x}\right|-2$ edges to $P\left(T_{n}\right)$ to quadrangulate the face bounded by $C_{x}, C_{w}$, and the attaching edges of $C_{w}$. Thus for every non-leaf node $x$ in $T_{n}$, chain $C_{x}$ is the left boundary of a face in $P\left(T_{n}\right)$ that can be quadrangulated to add $\left|C_{x}\right|-2$ extra faces to $P\left(T_{n}\right)$. We wish to count the number $F_{n}$ of such faces added:

$$
F_{n}=\sum_{\text {nonleaf nodes } x \text { in } T_{n}}\left(\left|C_{x}\right|-2\right) .
$$

Observe that if $x \in N_{n}(d)$, then $\left|C_{x}\right|=n-2 d$, since $\left|C_{0^{n}}\right|=n$ and for $y \neq 0^{n}$ in $T_{n}$, $\left|C_{p(y)}\right|=\left|C_{w}\right|+2$. Then

$$
F_{n}=\sum_{d=0}^{\left\lfloor\frac{n}{2}\right\rfloor}(n-2 d-2) \bar{L}_{n}(d) .
$$

Our goal is to show that $F_{n}=2^{n-1}-\left(\begin{array}{c}n \\ \left\lfloor\frac{n}{2}\right\rfloor\end{array}\right)$. We first need to be able to compute $\bar{L}_{n}(d)$.

Lemma 1 If $n>1$ and $0 \leq d \leq\left\lfloor\frac{n-1}{2}\right\rfloor$, then $\bar{L}_{n}(d)=\left(\begin{array}{c}n-1 \\ d\end{array}\right)-\left(\begin{array}{c}n-1 \\ d-1\end{array}\right)$, and 0 otherwise.

Proof. We show that $\bar{L}_{n}(d)=N_{n-1}(d)$ by showing that the mapping $f(x)=x 0$ is a bijection from the set of nodes in $T_{n-1}$ of weight $d$ to the set of non-leaves in $T_{n}$ of weight $d$. The result follows then from (2).

If $x$ is a node of weight $d$ in $T_{n-1}, x$ has no unmatched 1 , so neither does $x 0$, so $x 0$ is a node of weight $d$ in $T_{n}$. To see that $x 0$ is a nonleaf, note that since $2 d<n, x$ has an unmatched 0 , so $x 1$ is in $T_{n}$ and $p(x 1)=x 0$. Clearly $f$ is one-to-one. To see that $f$ is onto, let $y$ be a nonleaf node of weight $d$ in $T_{n}$. Then $y$ has a child $z$ and $z$ has the form $z=\alpha 10^{i}$ for some $i \geq 0$. Since $z$ is in $T_{n}, \alpha$ has no unmatched 1 and $y=p\left(\alpha 10^{i}\right)=\alpha 0^{i+1}$. Thus, $\alpha 0^{i}$ is in $T_{n-1}$ and $y=f\left(\alpha 0^{i}\right)$.

Lemma 2 For $n \geq 2$,

$$
\sum_{d=0}^{\left\lfloor\frac{n}{2}\right\rfloor} \bar{L}_{n}(d)=\left(\begin{array}{c}
n-1 \\
\left\lfloor\frac{n}{2}\right\rfloor-1
\end{array}\right) .
$$


Proof. Note that the summand is 0 if $d=\lfloor n / 2\rfloor$. Applying Lemma 1 gives a telescoping sum.

Lemma 3 For $n \geq 2$,

$$
\sum_{d=0}^{\left\lfloor\frac{n}{2}\right\rfloor} d \bar{L}_{n}(d)= \begin{cases}\frac{n}{2}\left(\begin{array}{c}
n-1 \\
\frac{n}{2}-1
\end{array}\right)-2^{n-2} & \text { if } n \text { is even } \\
\frac{n-1}{2}\left(\begin{array}{c}
n-1 \\
\left\lfloor\frac{n}{2}\right\rfloor-1
\end{array}\right)+\frac{1}{2}\left(\begin{array}{c}
n-1 \\
\left\lfloor\frac{n}{2}\right\rfloor
\end{array}\right)-2^{n-2} & \text { if } n \text { is odd. }\end{cases}
$$

Proof. First note that the summand is 0 when $d=0$ or $d=\left\lfloor\frac{n}{2}\right\rfloor$ and then apply Lemma 1 to get

$$
\begin{aligned}
\sum_{d=0}^{\left\lfloor\frac{n}{2}\right\rfloor} d \bar{L}_{n}(d) & =\sum_{d=1}^{\left\lfloor\frac{n}{2}\right\rfloor-1} d\left(\left(\begin{array}{c}
n-1 \\
d
\end{array}\right)-\left(\begin{array}{c}
n-1 \\
d-1
\end{array}\right)\right) \\
& =\sum_{d=1}^{\left\lfloor\frac{n}{2}\right\rfloor-1}\left(d\left(\begin{array}{c}
n-1 \\
d
\end{array}\right)-(d-1)\left(\begin{array}{c}
n-1 \\
d-1
\end{array}\right)\right)-\sum_{d=1}^{\left\lfloor\frac{n}{2}\right\rfloor-1}\left(\begin{array}{c}
n-1 \\
d-1
\end{array}\right) .
\end{aligned}
$$

The first sum telescopes, giving $\left(\left\lfloor\frac{n}{2}\right\rfloor-1\right)\left(\begin{array}{c}n-1 \\ \left\lfloor\frac{n}{2}\right\rfloor-1\end{array}\right)$. The second sum is the number of elements in the first $\left\lfloor\frac{n}{2}\right\rfloor-2$ levels of the Boolean lattice $\mathcal{B}_{n-1}$ which, when $n$ is even, is:

$$
\frac{1}{2}\left(2^{n-1}-2\left(\begin{array}{c}
n-1 \\
\left\lfloor\frac{n}{2}\right\rfloor-1
\end{array}\right)\right)
$$

and when $n$ is odd:

$$
\frac{1}{2}\left(2^{n-1}-2\left(\begin{array}{c}
n-1 \\
\left\lfloor\frac{n}{2}\right\rfloor-1
\end{array}\right)-\left(\begin{array}{c}
n-1 \\
\left\lfloor\frac{n}{2}\right\rfloor
\end{array}\right)\right)
$$

Theorem 4 For all $n \geq 2$, the number of faces in the plane graph $P\left(T_{n}\right)$ after quadrangulating is $2^{n-1}$. Thus, there are $2^{n-1}$ vertices in its dual, which is a Venn diagram for $n$ sets.

Proof. The embedding of the chain cover graph has $\left(\begin{array}{c}n \\ \lfloor n / 2\rfloor\end{array}\right)$ faces. We show that the number of such edges added in the quadrangulation phase is $F_{n}=2^{n-1}-\left(\begin{array}{c}n \\ \lfloor n / 2\rfloor\end{array}\right)$. By (4),

$$
F_{n}=\sum_{d=0}^{\left\lfloor\frac{n}{2}\right\rfloor}(n-2 d-2) \bar{L}_{n}(d)=(n-2) \sum_{d=0}^{\left\lfloor\frac{n}{2}\right\rfloor} \bar{L}_{n}(d)-2 \sum_{d=0}^{\left\lfloor\frac{n}{2}\right\rfloor} d \bar{L}_{n}(d) .
$$

Using Lemmas 2 and 3, when $n$ is even (7) becomes

$$
(n-2)\left(\begin{array}{c}
n-1 \\
\frac{n}{2}-1
\end{array}\right)+2^{n-1}-n\left(\begin{array}{c}
n-1 \\
\frac{n}{2}-1
\end{array}\right)=2^{n-1}-2\left(\begin{array}{c}
n-1 \\
\frac{n}{2}-1
\end{array}\right)=2^{n-1}-\left(\begin{array}{c}
n \\
\frac{n}{2}
\end{array}\right) .
$$


When $n$ is odd (7) becomes

$$
\begin{aligned}
(n-2)\left(\begin{array}{c}
n-1 \\
\left\lfloor\frac{n}{2}\right\rfloor-1
\end{array}\right)+2^{n-1}-(n-1)\left(\begin{array}{c}
n-1 \\
\left\lfloor\frac{n}{2}\right\rfloor-1
\end{array}\right)-\left(\begin{array}{c}
n-1 \\
\left\lfloor\frac{n}{2}\right\rfloor
\end{array}\right) & =2^{n-1}-\left(\begin{array}{c}
n-1 \\
\left\lfloor\frac{n}{2}\right\rfloor-1
\end{array}\right)-\left(\begin{array}{c}
n-1 \\
\left\lfloor\frac{n}{2}\right\rfloor
\end{array}\right) \\
& =2^{n-1}-\left(\begin{array}{c}
n \\
\left\lfloor\frac{n}{2}\right\rfloor
\end{array}\right) .
\end{aligned}
$$

This number is one more than half the number of vertices in a simple diagram of order $n$. Thus we propose to call these diagrams "half-simple".

\section{At Least Half-Simple Symmetric Diagrams for Prime $n$}

Recall that in Section 2, for prime $n$, the plane graph $R\left(P\left(S_{n}\right)\right)$ was created from $n$ copies of $P\left(S_{n}\right)$ and the dual of $R\left(P\left(S_{n}\right)\right)$ was a symmetric Venn diagram with $\left(\begin{array}{c}n \\ \lfloor n / 2\rfloor\end{array}\right)$ vertices. By Theorem 3, we can quadrangulate every face corresponding to a node and its first child in $S_{n}$ in every copy of $P\left(S_{n}\right)$ and the resulting quadrangulation of $R\left(P\left(S_{n}\right)\right)$ is still a plane, monotone, symmetric, spanning subgraph of the $n$-cube; thus its dual is still a symmetric Venn diagram. In this section we show that the total number of faces in the quadrangulation of $R\left(P\left(S_{n}\right)\right)$ is at least $2^{n-1}(1-o(1))$ and therefore its dual is asymptotically at least half-simple.

As an example for $n=11$ (see Figure 10), the number of faces of $P\left(S_{11}\right)$ before quadrangulating is $\left(\begin{array}{c}11 \\ 5\end{array}\right) / 11=42$. The number of faces added by quadrangulating the faces between the chains corresponding to a node in $S_{11}$ and its first child is 69 . Repeating this in every copy of $P\left(S_{11}\right)$, the total number of faces in the resulting quadrangulation of $R\left(P\left(S_{11}\right)\right)$ is $11(42+69)=1221$. In addition, note that any circular permutation can be used to label subsequent rotated copies of $P\left(S_{11}\right)$; if the circular permutation used is (6 789101112345 ), then 16 extra faces can be added to $P\left(S_{11}\right)$ by manually adding extra edges wherever possible, giving another $11 \times 16=171$ faces in the graph. Thus, the dual is a symmetric Venn diagram with $1221+171=1392$ vertices, whereas a simple Venn diagram would have 2046 vertices. This diagram is rigid, i.e. as simple as possible, as no more edges can be added to the dual graph and thus no more vertices can be separated in the Venn diagram.

For contrast, in [6], Hamburger shows how to separate vertices in his 11-Venn diagram from [7] to get symmetric 11-Venn diagrams with only up to 1001 vertices. Following his example, we can say that since each of the $69+16=85$ extra edges can either be present or not in the rotated copies of $P\left(S_{11}\right)$, these extra edges give us $2^{85}$ distinct symmetric 11-Venn diagrams, more than previously known.

It remains to count $F_{n}$, the number of faces added to $P\left(S_{n}\right)$ by quadrangulating the faces between the chains corresponding to a node in $S_{n}$ and its first child. Then $n F_{n}$ is the total number of faces added to $R\left(P\left(S_{n}\right)\right.$ ).

Recall from Section 2 that $x$ is a node in $S_{n}$ if and only if 


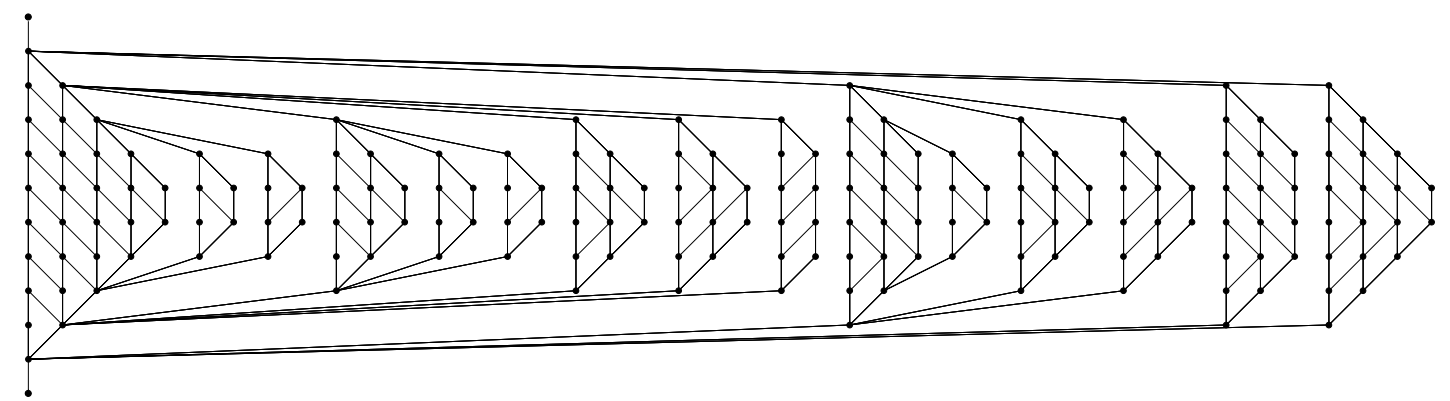

(a) After quadrangulating all faces corresponding to a node in $S_{11}$ and its first child.

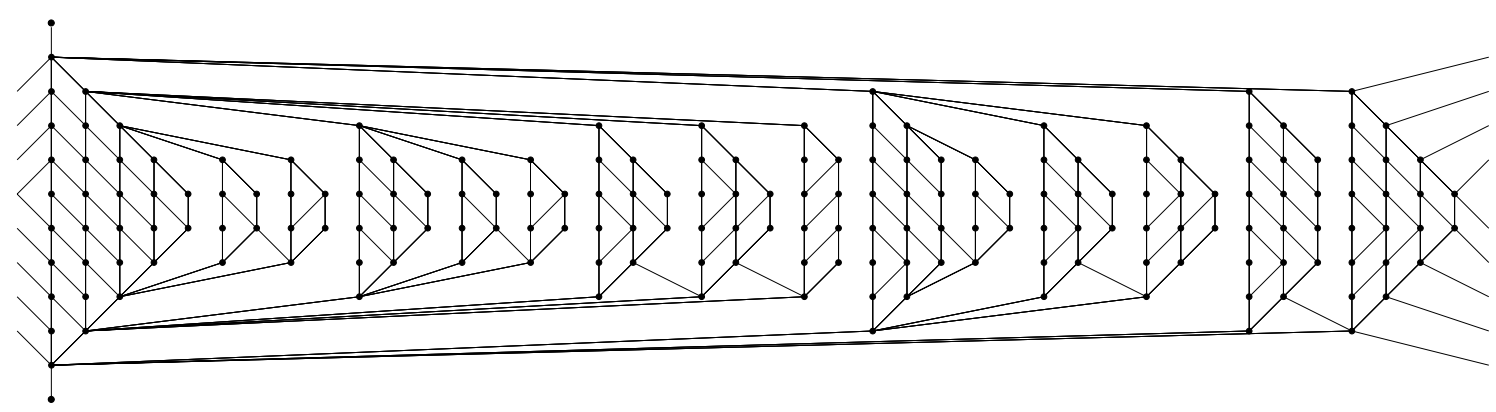

(b) After manually adding extra edges, including wrapping edges.

Figure 10: Adding edges to the graph $P\left(S_{11}\right)$. 
- (i) $x$ has finite block code (so it starts with 1 and ends with 0 ),

- (ii) $\beta(x) \leq \beta\left(\sigma^{i}(x)\right)$ for any $i$, and

- (iii) $x$ has exactly one unmatched 1 .

We will make use of the following lemma:

Lemma 4 If $x$ is a node in the chain cover tree and if the last block of $x$ has the form $10^{k}$, then $k \geq 2$.

Proof. First, $k \geq 1$ as $\beta(x)$ is finite. If $k=1$, then $\beta(x)=(\ldots, 2)$. However as $n=|x|$ is prime there must be an element in $\beta(x)$, call it $j$, such that $j \geq 3$ and so we could create a rotation of $x$ with a lexicographically smaller block code by rotating two positions right to create $x^{\prime}$ with $\beta\left(x^{\prime}\right)=(2, \ldots)$. This contradicts the fact that $x$ has the (unique) lexicographically lowest block code of all of its rotations.

Let $\ell(x)$ denote the location of the last 1 in $x$.

Theorem 5 If $u$ and $v$ are children of $w$ in the chain cover tree and $\ell(u)<\ell(v)$, then $v$ is not a leaf.

Proof. Since $w$ satisfies (i), $w$ can be written as

$$
w=\alpha 1^{a} 0^{b},
$$

where either $\alpha$ is empty or $\alpha$ has finite block code. Then $u$ and $v$ have the form

$$
u=\alpha 1^{a} 0^{c} 10^{b-c-1} ; \quad v=\alpha 1^{a} 0^{c^{\prime}} 10^{b-c^{\prime}-1},
$$

where $0 \leq c=\ell(u)-|\alpha|-a-1$ and $c<c^{\prime}=\ell(v)-|\alpha|-a-1$. Let

$$
z=\alpha 1^{a} 0^{c^{\prime}} 110^{b-c^{\prime}-2} .
$$

If we show that $z$ satisfies (i)-(iii), then $z$ is a child of $v$ in the chain cover tree.

Applying Lemma 4 to $v$ gives that $b-c^{\prime}-2 \geq 2$, so $z$ ends in 0 and starts with 1 , satisfying (i). Also $\beta(z)=\beta(v)$, so $z$ satisfies (ii). Finally, since $u$ is a node, it satisfies (iii) and therefore the following string has exactly one unmatched 1 , which, since $c \geq 1$, is not the last 1 :

$$
\alpha 1^{a} 0^{c} 1 .
$$

Then, since $c^{\prime}>c$, the string

$$
\alpha 1^{a} 0^{c^{\prime}} 1
$$

has exactly one unmatched 1 and at least one unmatched 0 , so the string

$$
\alpha 1^{a} 0^{c^{\prime}} 11
$$

(and therefore also $z$ ) has exactly one unmatched 1. So $z$ satisfies (iii). 
Corollary 1 Each node in the chain cover tree has at most one leaf child.

We make the following definitions.

- Let $N(d)$ denote the number of nodes of weight $d$ in $S_{n}$. Then $N(1)=1$ and for $d>1$,

$$
N(d)=\frac{1}{n}\left[\left(\begin{array}{l}
n \\
d
\end{array}\right)-\left(\begin{array}{c}
n \\
d-1
\end{array}\right)\right],
$$

- Let $L(d)$ denote the number of leaves of weight $d$ in $S_{n}$.

- Let $\bar{L}(d)$ denote the number of nonleaves of weight $d$ in $S_{n}$.

- Let $w(d)$ denote the number of faces added to $P\left(S_{n}\right)$ by quadrangulating the region between the chains corresponding to a node $x$ of weight $d$ its first child $w$ in $S_{n}$. From Theorem 2 we know that

$$
w(d)=\left|C_{w}\right|=\left|C_{x}\right|-2=n-2 d-2 \text { for } 1 \leq d \leq(n-3) / 2 .
$$

Then $F_{n}$, the number of faces added to $P\left(S_{n}\right)$, can be expressed as

$$
F_{n}=\sum_{d=1}^{(n-3) / 2} w(d) \bar{L}(d)
$$

We can get a lower bound on $F_{n}$ by observing first that every node at level $d$ contributes either $w(d-1)$ toward quadrangulating a region with its parent (if it is a leaf) or $w(d)$ toward quadrangulating a region with its first child (if it is a nonleaf) or both. By Corollary 1, leaves can be mapped one-to-one to parents. Thus if we count all of the contributions at every node at every level, we are at worst double-counting, giving the second inequality below. The first inequality follows since $w(d) \leq w(d-1)$ and $N(d)=$ $L(d)+\bar{L}(d)$.

$$
\sum_{d=1}^{(n-3) / 2} w(d) N(d) \leq \sum_{d=1}^{(n-3) / 2}\left(w(d-1) L(d)+w(d) \bar{L}(d) \leq 2 \sum_{d=1}^{(n-3) / 2} \bar{L}(d) w(d) .\right.
$$

Thus, the number of faces added to $R\left(P\left(S_{n}\right)\right)$ by quadrangulating is

$$
n F_{n} \geq \frac{n}{2} \sum_{d=1}^{(n-3) / 2} w(d) N(d) .
$$

Theorem 6 For $n$ prime, the number of faces added to $R\left(P\left(S_{n}\right)\right)$ by quadrangulating is

$$
n F_{n} \geq 2^{n-1}-1-\frac{1}{2}\left[\left(\begin{array}{c}
n+1 \\
(n+1) / 2
\end{array}\right)+\left(\begin{array}{c}
n \\
(n-3) / 2
\end{array}\right)\right],
$$

making its dual a symmetric Venn diagram with $2^{n-1}(1-o(1))$ vertices. 
Proof. We need to show that (11) is a lower bound for (10).

We will make use of the following identity, established in the proof of Theorem 4, with the observation that in $(7), \bar{L}\left(\left\lfloor\frac{n}{2}\right\rfloor\right)=0$.

$$
\sum_{d=0}^{\left\lfloor\frac{n}{2}\right\rfloor-1}(n-2 d-2)\left[\left(\begin{array}{c}
n-1 \\
d
\end{array}\right)-\left(\begin{array}{l}
n-1 \\
d-1
\end{array}\right)\right]=2^{n-1}-\left(\begin{array}{c}
n \\
\lfloor n / 2\rfloor
\end{array}\right) .
$$

If we replace $n$ by $n+1$ and observe that the summand on the left is $n-1$ when $d=0$ then for odd $n$, we get

$$
\sum_{d=1}^{(n+1) / 2-1}(n-2 d-1)\left[\left(\begin{array}{l}
n \\
d
\end{array}\right)-\left(\begin{array}{c}
n \\
d-1
\end{array}\right)\right]=2^{n}-\left(\begin{array}{c}
n+1 \\
(n+1) / 2
\end{array}\right)-(n-1) .
$$

Starting from (10) and using (8) and (9),

$$
\begin{aligned}
\frac{n}{2} \sum_{d=1}^{(n-3) / 2} w(d) N(d) & =\frac{n}{2}\left((n-4)+\sum_{d=2}^{(n-3) / 2}(n-2 d-2) \frac{1}{n}\left[\left(\begin{array}{l}
n \\
d
\end{array}\right)-\left(\begin{array}{c}
n \\
d-1
\end{array}\right)\right]\right) \\
& =\frac{1}{2}\left(n(n-4)-(n-1)(n-4)+\sum_{d=1}^{(n-3) / 2}(n-2 d-2)\left[\left(\begin{array}{l}
n \\
d
\end{array}\right)-\left(\begin{array}{c}
n \\
d-1
\end{array}\right)\right]\right) \\
& =\frac{1}{2}\left(n-4+\sum_{d=1}^{(n-3) / 2}(n-2 d-1)\left[\left(\begin{array}{l}
n \\
d
\end{array}\right)-\left(\begin{array}{c}
n \\
d-1
\end{array}\right)\right]\right. \\
& =\frac{1}{2}\left(n-4+\sum_{d=1}^{(n-3) / 2}(n-2 d-1)\left[\left(\begin{array}{c}
n \\
d
\end{array}\right)-\left(\begin{array}{c}
n \\
d-1
\end{array}\right)\right]-\left(\begin{array}{c}
n \\
(n-3) / 2
\end{array}\right)+1\right) .
\end{aligned}
$$

We now make use of (13) to evaluate the sum in the last line above, noting that the last term in the sum on the left of (13) is 0 :

$$
\begin{aligned}
\frac{n}{2} \sum_{d=1}^{(n-3) / 2} w(d) N(d) & =\frac{1}{2}\left(n-4+2^{n}-\left(\begin{array}{c}
n+1 \\
(n+1) / 2
\end{array}\right)-(n-1)-\left(\begin{array}{c}
n \\
(n-3) / 2
\end{array}\right)+1\right) \\
& =2^{n-1}-1-\frac{1}{2}\left(\left(\begin{array}{c}
n+1 \\
(n+1) / 2
\end{array}\right)+\left(\begin{array}{c}
n \\
(n-3) / 2
\end{array}\right)\right) .
\end{aligned}
$$

This lower bound asymptotically approaches $2^{n-1}$ as $n$ increases.

In fact, the number of faces resulting from the quadrangulations appears to be considerably larger than the lower bound given by the theorem, but we have been unable to 
find a tighter counting argument which would establish this. The table below shows a comparison of the number of vertices in a simple Venn diagram, the number of vertices in the symmetric Venn diagrams produced by the GKS construction, the number of vertices in the symmetric Venn diagrams produced by the construction of this paper (KRSW), and the ratio of the number of vertices produced by the KRSW construction to the number in a simple Venn diagram.

\begin{tabular}{|r|r|r|r|c|}
\hline$n$ & simple & {$[$ GKS $]$} & {$[$ KRSW $]$} & $\frac{[\text { KRSW }]}{\text { simple }}$ \\
\hline 3 & 6 & 3 & 3 & .5000 \\
\hline 5 & 30 & 10 & 15 & .5000 \\
\hline 7 & 126 & 35 & 70 & .5556 \\
\hline 11 & 2,046 & 462 & 1,221 & .5968 \\
\hline 13 & 8,190 & 1,716 & 5,005 & .6111 \\
\hline 17 & 131,070 & 24,310 & 81,787 & .6240 \\
\hline 19 & 524,286 & 92,378 & 329,289 & .6281 \\
\hline 23 & $8,388,606$ & $1,352,078$ & $5,308,423$ & .6328 \\
\hline
\end{tabular}

\section{Concluding Remarks}

We have succeeding in showing that for prime $n$ there are symmetric Venn diagrams that are nearly simple, in the sense that the average number of curves passing through a point of intersection is at most a constant $c$, independent of $n$. Although we have established this for (asymptotically) $c=4$, the same construction with an improved counting argument could likely lower the constant $c$. The question remains as to whether there are simple symmetric Venn diagrams for $n$ prime and greater than 7 ; even the the $n=11$ case remains open.

It would also be interesting to prove an upper bound on the number of symmetric Venn diagrams.

\section{References}

[1] Bette Bultena, Branko Grünbaum, and Frank Ruskey. Convex drawings of intersecting families of simple closed curves. In 11th Canadian Conference on Computational Geometry, pages 18-21, 1999.

[2] Bette Bultena and Frank Ruskey. Venn diagrams with few vertices. Electron. J. Combin., 5(1):Research Paper 44, 21 pp. (electronic), 1998.

[3] Curtis Greene and Daniel J. Kleitman. Strong versions of Sperner's theorem. J. Combinatorial Theory Ser. A, 20(1):80-88, 1976.

[4] Jerrold Griggs, Charles E. Killian, and Carla D. Savage. Venn diagrams and symmetric chain decompositions in the Boolean lattice. Electron. J. Combin., 11:Research Paper 2, 30 pp. (electronic), 2004. 
[5] Branko Grünbaum. Venn diagrams and independent families of sets. Math. Mag., 48:12-23, 1975 .

[6] Peter Hamburger. Pretty drawings. More doodles and doilies, symmetric Venn diagrams. Utilitas Mathematica. To appear, preprint (http://www.ipfw.edu/math/hamburger/papers.htm).

[7] Peter Hamburger. Doodles and doilies, non-simple symmetric Venn diagrams. Discrete Math., 257(2-3):423-439, 2002.

[8] D. W. Henderson. Venn diagrams for more than four classes. American Mathematical Monthly, 70:424-426, 1963.

[9] Frank Ruskey. A survey of Venn diagrams. Electron. J. Combin., 4(1):Dynamic Survey 5 (electronic), 2001. 